OPEN ACCESS

Edited by:

Kamalakannan Palanichamy,

The Ohio State University,

United States

Reviewed by:

Güliz Acker,

Charité Medical University of Berlin,

Germany

Michele Solimena,

Technische Universität Dresden,

Germany

*Correspondence:

Hao Zhuang

zhh8764@163.com

Budong Chen

cbdgby@163.com

Jie Zhuo

zhuojietj@outlook.com

Bo Wang

dr.bo.wang@tmu.edu.cn

${ }^{t}$ These authors have contributed equally to this work

Specialty section:

This article was submitted to

Neuro-Oncology and

Neurosurgical Oncology,

a section of the journal

Frontiers in Oncology

Received: 05 March 2021

Accepted: 09 July 2021

Published: 07 September 2021

Citation:

Wang D, Tang F, Liu X, Fan Y, Zheng $Y$, Zhuang $H$, Chen $B$, Zhuo J and Wang $B$ (2021) Expression and Tumor-Promoting Effect of Tyrosine

Phosphatase Receptor Type N

(PTPRN) in Human Glioma.

Front. Oncol. 11:676287.

doi: 10.3389/fonc.2021.676287

\section{Expression and Tumor-Promoting Effect of Tyrosine Phosphatase Receptor Type N (PTPRN) in Human Glioma}

\author{
Dong Wang ${ }^{1 \dagger}$, Fan Tang ${ }^{2 \dagger}$, Xi Liu $^{3 \dagger}$, Yueshan Fan ${ }^{1}$, Yu Zheng ${ }^{4}$, Hao Zhuang ${ }^{5 *}$, \\ Budong Chen ${ }^{6,7,8^{*}}$, Jie Zhuo ${ }^{6,7,8^{*}}$ and Bo Wang ${ }^{6,7,8^{*}}$
}

${ }_{1}^{1}$ Department of Neurosurgery, Tianjin Medical University, General Hospital, Tianjin Key Laboratory of Injuries, Variations, and Regeneration of Nervous System, Tianjin Neurological Institute, Tianjin, China, ${ }^{2}$ Department of Pathology, Tianjin Huanhu Hospital, Tianjin Key Laboratory of Cerebral Vascular and Neurodegenerative Diseases, Tianjin Neurosurgical Institute, Tianjin, China, ${ }^{3}$ Department of Gastroenterology, Tianjin Nankai Hospital, Tianjin, China, ${ }^{4}$ GCP Center, Tianjin Medical University Cancer Institute \& Hospital, Tianjin, China, ${ }^{5}$ Department of Hepatic Biliary Pancreatic Surgery, Cancer Hospital Affiliated to Zhengzhou University, Zhengzhou, China, ${ }^{6}$ Department of Neurosurgery, Tianjin Huanhu Hospital, Tianjin Key Laboratory of Cerebral Vascular and Neurodegenerative Diseases, Tianjin Neurosurgical Institute, Tianjin, China, ${ }^{7}$ Department of Neurosurgery, Tianjin University Huanhu Hospital, Tianjin, China, ${ }^{8}$ Department of Neurosurgery, Huanhu Hospital Affiliated to Nankai University, Tianjin, China

Tyrosine phosphatase receptor type N (PTPRN) plays an important role in the regulation of the secretion pathways of various neuroendocrine cells. Moreover, PTPRN was demonstrated to play a crucial role in the initiation and progression of the signalling cascade regulating cell function. In this study, fifty-seven glioma patients were enrolled for clinical and prognostic analyses. The cell phenotype was determined by cell proliferation and migration assays. RNA-seq, co-IP and mass spectrometry were used to study the molecular mechanism of the effects of PTPRN on cell proliferation and metastasis. The result showed that High expression of PTPRN indicated a poor prognosis of high-grade glioma. PTPRN downregulation reduced the proliferation and migration of glioma cells, and PTPRN overexpression induced the proliferation and migration of glioma cells. PTPRN knockdown decreased tumor growth in a mouse xenograft model. Effect of PTPRN knockdown on the transcriptome was studied in U87 glioma cells. PTPRN activated the PI3K/AKT pathway by interacting with HSP90AA1. In conclusion, PTPRN is an important proliferation- and metastasis-promoting factor. Reducing the expression of PTPRN in glioma cells can be used as a potential therapeutic strategy.

Keywords: PTPRN, glioma, RNA-seq, GSEA, Akt

\section{INTRODUCTION}

Glioma accounts for approximately 50\% of all malignant brain tumors (1). Prognosis of glioma patients is related to the volume, WHO grade and location of the tumor. Currently, radiotherapy, chemotherapy, gene therapy and other treatment methods are mainly used in the clinic. However, due to chemoradiotherapy resistance, the median survival time of high-grade glioma patients is less 
than 12 months (2). Therefore, urgent investigations of the molecular mechanism of occurrence and development of gliomas and exploration of effective methods for the diagnosis and treatment of glioma are needed.

Protein tyrosine phosphatase receptor type N (PTPRN) is located on human chromosome band $2 \mathrm{q} 35$. PTPRN is mainly expressed in endocrine cells, neurons of autonomic nervous system and neuroendocrine neurons of brain, including pancreas, pituitary, adrenal medulla, amygdala and hypothalamus, because they all contain neurosecretory granules $(3,4)$. PTPRN is a type I transmembrane protein that has an inactive protein tyrosine phosphatase (PTP) domain (5). PTPRN participates in neuroendocrine processes, such as biogenesis, transport and/or regulation of exocytosis (6). Torii $S$ et al. demonstrated that PTPRN has an important effect on the regulation of the secretion pathways in various neuroendocrine cells, possibly by regulating hormone content (7). Diabetes-related gene screening experiments indicated that PTPRN plays an important role in occurrence and development of diabetes mellitus (8-10). Mziaut $\mathrm{H}$ demonstrated that PTPRN increases secretory granule gene transcription by binding to STAT5 (11).

We analysed the prognosis of glioma patients and observed the effect of PTPRN on the phenotype of glioma cells. To clarify the mechanism of action of PTPRN in glioma cells, we downregulated PTPRN, detected the changes in the pathways in glioma cells, and determined possible factors related to PTPRN that influenced the changes in the pathways.

\section{MATERIALS AND METHODS}

\section{Clinical Specimens}

Retrospective analysis of 57 patients with glioma. The patients did not undergo any treatment before surgery. The control brain tissue of 5 cases (cerebral cortex) were from the decompressor brain tissue which were removed by trauma patients. All clinical tissue samples were taken from Tianjin Huanhu hospital. All the specimens were obtained with the consent of the ethics committee of Tianjin Huanhu hospital and the consent of the patients' family members. The WHO grades of glioma patients were assigned by two pathologists, and they were followed up for 3 years.

\section{The Cancer Genome Atlas (TCGA) Dataset}

TCGA database was analyzed by "Gene Expression Profiling Interactive Analysis (GEPIA)" and "The Human Protein Atlas". GEPIA provides key interactive analysis and customization functions, including tumor/normal differential expression profile analysis, profiling, pathological staging, patient survival analysis, similar gene detection analysis, and dimensionality reduction analysis. GEPIA was used to analyze the PTPRN expression in GBM of TCGA, and the survival analysis module was used. The screening conditions were as follows, method: OS, group cut off: median, hazards ratio: yes, 95\% confidence interval: yes, axis units: months; data sets selection: GBM. The Human Protein Atlas is to study the immunohistochemical staining status of proteins in human normal tissues, cancer tissues, and cancer cell lines. It can be used to analyze the differential expression of proteins in tumor tissues and to analyze the survival of genes and tumors. The screening conditions were as follows: pathology, prognostic summary, cut off: median.

\section{Immunohistochemical Analysis}

Immunohistochemistry was used to detect the expression of PTPRN, vascular endothelial growth factor (VEGF), and O6methylguanine-DNA methyltransferase (MGMT). The operation steps were strictly in accordance with the instructions of the kit. $4 \mu \mathrm{M}$ thick sections were dewaxed with xylene. Gradient ethanol dehydration. Citrate buffer $(\mathrm{pH}=6.0)$ was used for antigen repair in a pressure cooker. After cooling, PBS was washed 3 times. First antibody: anti-PTPRN antibody (ab207750, 1:200, Abcam, UK), anti-VEGF antibody (EP1176Y, 1:200, Zhongshan, China), anti-MGMT antibody (UMAB56, 1:200, Zhongshan, China), were added and incubated at $4^{\circ} \mathrm{C}$ overnight. After the first antibody was rewarming and washed with PBS, the second antibody (DS9800, Leica, German) was added and incubated at room temperature for $20 \mathrm{~min}$. DAB stained, hematoxylin stained, and neutral resin was used to seal the sections.

Each section was determined by two senior pathologists according to the staining proportion and intensity of positive cells. According to the staining intensity, no staining score was 0 , the light yellow (weak positive) score was 1, the brown-yellow (medium positive) score was 2 , and the brown (strongly positive) score was 3. According to the staining proportion of positive cells, the score of no positive tumor cells was 0 , the score of positive cells less than $20 \%$ was 1 , the score of $20 \%$ to $50 \%$ was 2, the score of $51 \%$ to $70 \%$ was 3 , and the score of more than $70 \%$ was 4 . Multiply the above two scores as the final result.

\section{Cell Culture and Transfection}

We used the U87 and U251 glioma cell lines (ATCC) in the present study. The cells were cultured in a DMEM medium containing $10 \%$ FBS at $37^{\circ} \mathrm{C}$ and $5 \% \mathrm{CO}_{2}$. The cells were transfected when they reached the logarithmic growth phase. For cell transfection to downregulate and upregulate PTPRN, short-hairpin RNA and overexpression plasmids were used, respectively. The oligonucleotides targeting PTPRN were ligated to $\mathrm{pLKO} .1$ vector, following the procedure of Addgene, and the vector with a similar GC ratio and no random sequence matched with any known human coding gene was ligated as the control group. The overexpression plasmid was constructed using cDNA of the human U87 glioma cell line as a template. PCR products were ligated to the TA clone vector PCR8/GW/TOPO (Invitrogen, USA). The recombinant plasmid containing the correct fragment was recombined with lentivirus target plasmid by LR clonase using gateway method. (Supplementary Table 1).

\section{Reverse Transcription-Quantitative Polymerase Chain Reaction (RT-qPCR)}

Total RNA was extracted with RNase-free reagent. TRIzol (Invitrogen, USA) was used for RNA isolation, which was followed by RNA reverse transcription. RNA precipitation was dissolved in pure water without RNase, and the concentration of RNA was determined by spectrophotometer (A260/280). RT-qPCR 
was performed using an SYBR kit (Promega, USA), and the results were calculated by the $2^{-\triangle \triangle \mathrm{Ct}}$ method. The primers for PTPRN and GAPDH used in this study are shown in the Supplementary File.

\section{Western Blot}

The protein was extracted with RIPA lysis buffer, and the concentration of the protein was assayed by the BCA method. Proteins were denatured at $100^{\circ} \mathrm{C}$ for $15 \mathrm{~min}$ and separated by $10 \%$ SDS-PAGE. PVDF membranes were blocked with 5\% BSA (Solarbio, China) and incubated with primary antibodies against PTPRN (ab207750, 1:1000, Abcam, UK), E-cadherin (3195S, 1:1000, CST, USA), N-cadherin (13116S, 1:1000, CST, USA), Snail (3879S, 1:1000, CST, USA), HSP90AA1(8165S, 1:1000, Abcam, UK), AKT (3195S, 1:1000, CST, USA), p-AKT (4060S, 1:1000, CST, USA), mTOR (2983S, 1:1000, CST, USA), p-mTOR (2974S, 1:1000, CST, USA), GAPDH (5174S, 1:1000, CST, USA) and secondary antibodies (7074S, 1:2000, CST, USA). The expression of these proteins was visualized by using an enhanced chemiluminescence kit (Merck, USA).

\section{Cell Counting Kit-8 Assay}

The cell counting kit-8 (CCK-8, Beyotime, China) assay was used to measure the proliferation of the cells. In 96 wells plate, $1 \times 10^{3}$ cells were added into each well, and each group of cells was set with 3 multiple wells. When testing was needed, a CCK-8 solution was added. After incubation in an incubator for $2 \mathrm{~h}$, the absorbance value at $450 \mathrm{~nm}$ was detected by spectrophotometer. The corresponding OD values were detected at $24 \mathrm{~h}, 48 \mathrm{~h}, 72 \mathrm{~h}$, and $96 \mathrm{~h}$. The experiment was repeated three times.

\section{Colony Formation Assay}

The colony formation assay was used to measure the proliferation of the cells. In 96 wells plate, cells transfected with PRPTN-shRNA and PTPRN plasmids were cultured for 14 days under standard conditions at $1 \times 10^{3}$ cells/well. Then, the colonies were fixed with $4 \%$ paraformaldehyde and stained with $0.5 \%$ crystal violet. The results were recorded using a camera. The experiment was repeated three times.

\section{Wounding Healing Assay}

The wounding healing assay was used to measure the migration of the cells. Cells transfected with PRPTN-shRNA and PTPRN plasmids were cultured in 6-well plates. The cells at a density of $5 \times 10^{4} /$ well were scratched with a pipette tip. Then, the cells were cultured in a serum-free medium for $24 \mathrm{~h}$. The scratch was imaged at 0 and $24 \mathrm{~h}$. The area of scratch reduction was calculated. The experiment was repeated three times.

\section{Extreme Limiting Dilution Analysis}

The extreme limiting dilution analysis was used to measure the self-renewal ability of glioma stem cells. Cells transfected with PRPTN-shRNA and PTPRN plasmids were cultured in DMEM/ F12 with B27 (Gibco, USA), insulin, EGF, and bFGF (Sino, China) for 14 days. The glioma stem cell (GSC) frequency was calculated by using extreme limiting dilution analysis (ELDA, bioinf.wehi.edu.au/software/elda/).

\section{RNA-Sequencing}

The efficiency of transfection was verified by reverse transcription-quantitative polymerase chain reaction. The U87 cells and PTPRN knockdown U87 cells were lysed with TRIzol. Total RNA was extracted with RNase-free reagent. RNA-seq was performed by BGI Company (Shenzhen, China) using BGISEQ500. GEO Series record number was GSE175831. We also carried out gene set enrichment analysis (GSEA, USA) and pathway enrichment analysis.

\section{Co-Immunoprecipitation (Co-IP) and Liquid Chromatography-Tandem Mass Spectrometry (LC-MS/MS) Analysis}

Transfected 293T or U87 cells were collected, and lysis buffer (20 $\mathrm{mM}$ Tris- $\mathrm{HCl}$ ( $\mathrm{pH} \mathrm{8),} 137 \mathrm{mM} \mathrm{NaCl}, 0.5 \%$ Triton X-100, and 2 $\mathrm{mM}$ EDTA) and protease inhibitor cocktail were added at $4^{\circ} \mathrm{C}$ for $30 \mathrm{~min}$. The mixture was centrifuged at 15,000 rpm for 30 min. Part of the supernatant for Co-IP was incubated with a primary anti-FLAG antibody (Sigma-Aldrich, USA) overnight. The agarose slurry was added and slowly shaken for $4 \mathrm{~h}$ at $4^{\circ} \mathrm{C}$. Then, the agarose slurry was collected, and the adsorbed protein was washed and collected for LC-MS/MS analysis (Shanghai Applied Protein Technology) and western blot analysis.

\section{Animal Experiments}

The animal experiment design was approved by the animal experiment ethics review committee of Tianjin Huanhu hospital. All operations and experimental procedures are in accordance with the regulations on the management of laboratory animals. The experimental animals were reared in the SPF animal laboratory of the experimental animal center of Tianjin Medical University. Twelve 5-7 weeks old athymic nude mice weighing 17-20 g (Huafukang Biotechnology, China) were randomly divided into the control group and PTPRN knockdown group. The nude mice were anesthetized and placed in a single-arm digital display stereotactic device. A skull drill was drilled $1 \mathrm{~mm}$ in front of the anterior fontanel and $2 \mathrm{~mm}$ on the right side of the midline. Then, $1 \times 10^{5}$ cells were injected into the brain. After that, the motor ability and neurological signs of the mice were observed every 3 days and weighed once a week. The nude mice were euthanized when they showed signs of cachexia or neurological dysfunction. The mice were imaged for fluc activity by a bioluminescence imaging system (IVIS ${ }^{\circledR}$ Lumina III, USA) and examined by magnetic resonance imaging (MRI, Siemens, German).

\section{Statistical Analysis}

The data of the study were analysed by SPSS 16.0 (Chicago, IL, USA). The data are presented as the mean \pm SD. The clinical data were analysed by ANOVA. The survival of patients and nude mice was analysed by the Kaplan-Meier method. The T test was used for comparison of two groups. $\mathrm{p}<0.05$ indicated that the results are statistically significant. 


\section{RESULTS}

\section{High Expression of PTPRN Indicated a Poor Prognosis of High-Grade Glioma}

Survival analysis of PTPRN expression based on the TCGA database showed that high expression of PTPRN indicated a poor prognosis of high-grade glioma (Figure 1B). Our clinical and experimental results were consistent with the database analysis results (Figure 1C). The expression level of PTPRN was divided into a low (score 0-4) and high (score 6-12) expression group using the mean expression score 5.28 as a cutoff point (Figure 1A). The overall survival (OS) of patients with high PTPRN expression was shorter than that of patients with low PTPRN expression. Univariate analysis showed that the level of PTPRN, MGMT, VEGF and WHO grade were associated with the prognosis of glioma patients. Moreover, the expression of PTPRN was an independent factor for the prognosis of glioma patients (Figure 1D). In addition, we found that there was a positive correlation between PTPRN and MGMT; however, there was no association between the level of PTPRN and the other clinicopathological indexes, which include sex, age, Karnofsky Performance Status (KPS), tumor diameter, VEGF (Table 1).
A
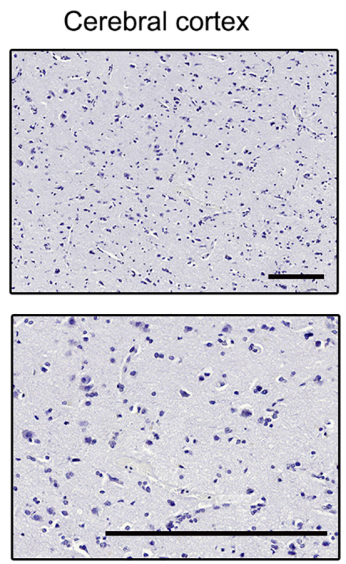

Low PTPRN expression
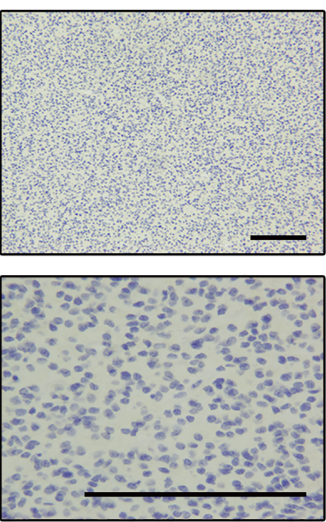

High PTPRN expression
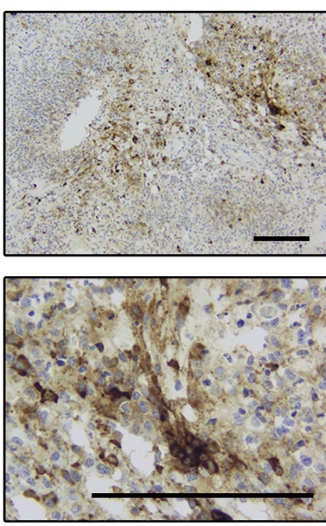

B

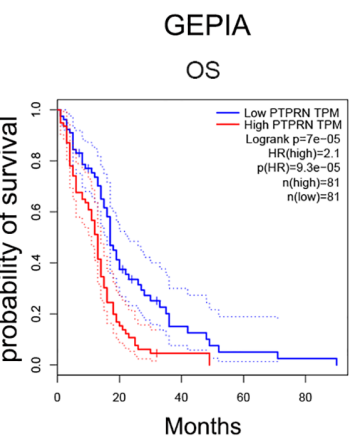

The human protein atlas

os

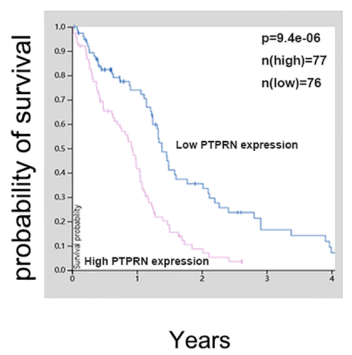

D

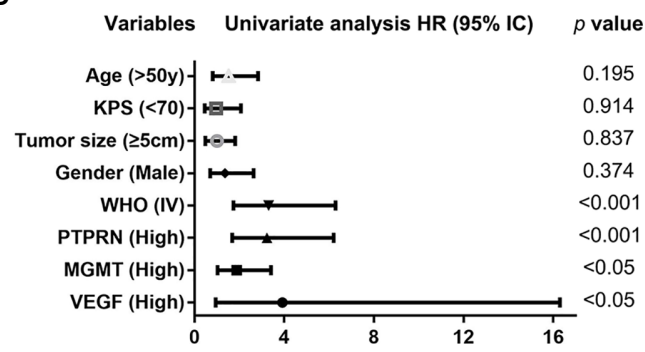

C

PFS

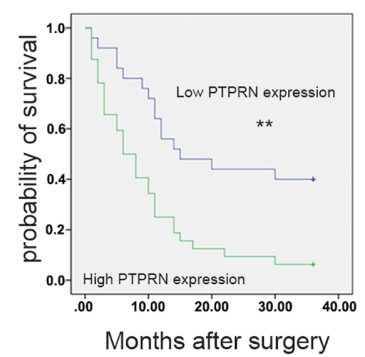

os

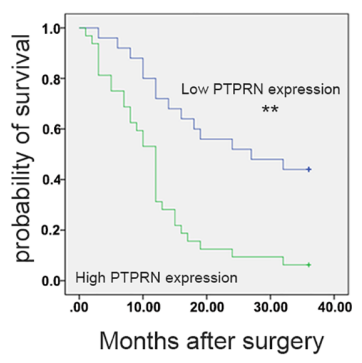

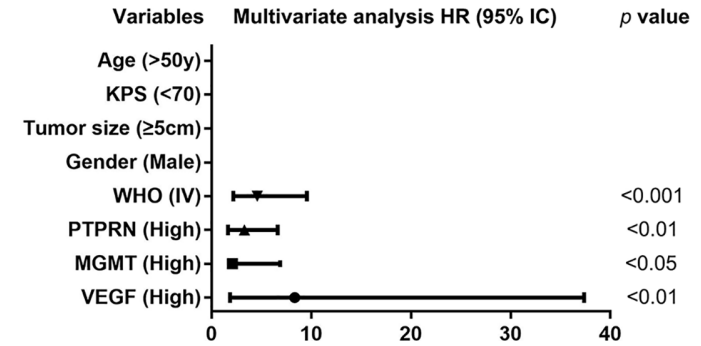

FIGURE 1 | High expression of PTPRN indicated a poor prognosis of high-grade glioma. (A) PTPRN expression detected by immunohistochemistry staining in cerebral cortex tissue (negative) and glioblastoma (low and high PTPRN expression); (B) Survival analysis of PTPRN expression based on the GEPIA and Human Protein Atlas database (log-rank test); (C) Survival analysis of PTPRN expression in fifty-seven high-grade glioma patients (log-rank test); (D) Univariate and multivariate survival analysis between PTPRN expression and clinicopathological parameters; scale bars, $100 \mu \mathrm{m}$; ${ }^{* *} \mathrm{p}<0.01$. 
TABLE 1 | The relationship between PTPRN expression with clinicopathological features.

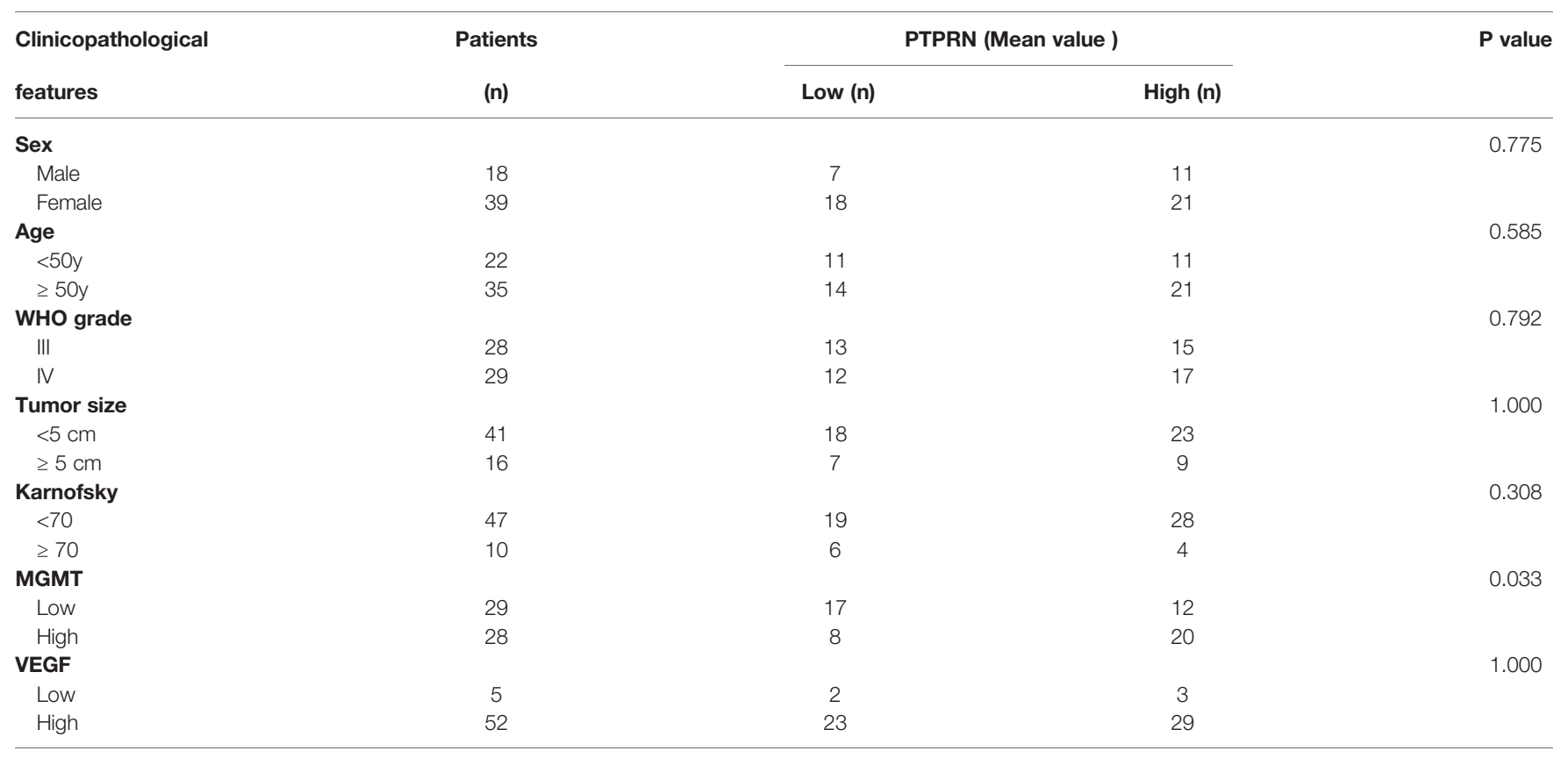

\section{PTPRN Downregulation Reduced the Proliferation and Migration of Glioma Cells}

The level of PTPRN was measured by RT-qPCR and western blot analysis after the transfection with PTPRN-shRNA (Figures 2A, B). The results of CCK8 and colony formation assays showed that knockdown of PTPRN reduced the proliferation of U87 cells (Figures 2C, D). The migration was inhibited in U87 cells with low expression of PTPRN (Figure 2E). The glioma stem cell frequency was decreased in the PTPRN knockdown group (Figure 2F). The detection of epithelial mesenchymal transition (EMT)-related markers by western blot analysis showed that $\mathrm{N}$ cadherin and Snail expression followed the trend of PTPRN. However, the level of E-cadherin was increased by knockdown of PTPRN (Figure 2G).

\section{PTPRN Overexpression Induced the Proliferation and Migration of Glioma Cells}

The results of MTS and colony formation assays demonstrated that upregulation of PTPRN induced the proliferation of U251 cells (Figures 3A-D). The migration was induced in U251 cells with high expression of PTPRN (Figure 3E). The glioma stem cell frequency was decreased in the PTPRN overexpression group (Figure 3F). The detection of epithelial mesenchymal transition (EMT)-related markers by western blot analysis showed that N-cadherin and Snail expression followed the trend of PTPRN. However, the level of E-cadherin was decreased after overexpression of PTPRN (Figure 3G).

\section{Effect of PTPRN Knockdown on the Transcriptome of U87 Glioma Cells}

RNA-seq was conducted to confirm the pathways that played a role in PTPRN functions in glioma. Fold change $\geq 2.0$ and FDR $\leq 0.001$ were used to identify the differential genes
(Figure 4A). Then, the KEGG method was used to analyze the differential genes. PI3K/AKT pathway was also associated with the changes in PTPRN (Figure 4B). The number of genes with altered expression was shown by Volcano plot analysis (Figure 4C). We conducted Gene Ontology analysis, and differential genes were categorized into three groups, including biological process, cellular component and molecular function (Figure 4D). GSEA showed that the PIK3/AKT pathways were related to the changes in PTPRN (Figure 4E).

\section{PTPRN Knockdown Decreased Tumor Growth in a Mouse Xenograft Model}

The volume of the tumor was measured by bioluminescence imaging (Figure 5A). The tumor volume of PTPRN knockdown mice was significantly decreased (Figure 5B). MRI also showed knockdown of PTPRN repressed the growth of the tumors (Figure 5C). Kaplan-Meier survival curves was used to analysis the difference of prognosis between control group and PTPRN knockdown group. The survival in the mouse xenograft model was improved by downregulation of PTPRN (Figure 5D).

\section{PTPRN Activated the PI3K/AKT Pathway by Interacting With Heat Shock Protein 90 Alpha Family Class A Member 1 (HSP90AA1)}

An interaction between PTPRN and HSP90AA1 was identified by Co-IP and LC-MS/MS in FLAG-HA-PTPRN-expressing $293 \mathrm{~T}$ cells (Figure 6A). Immunoprecipitation and western blot analysis demonstrated that PTPRN interacted with HSP90AA1 in U87 cells (Figure 6B). PTPRN regulated the PI3K/AKT pathway activity. BEZ235 was used to reverse the changes in the activity of the PI3K/AKT pathway in U251 cells induced by 
A

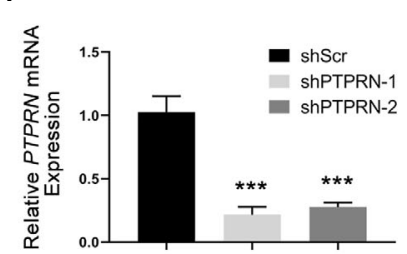

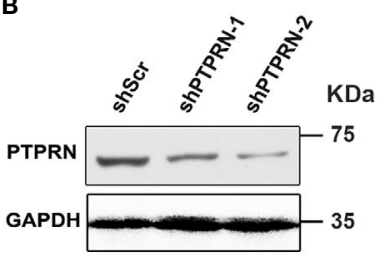

D

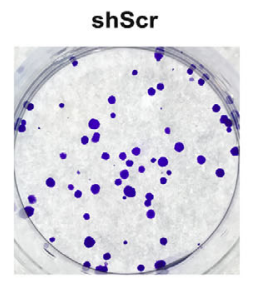

E
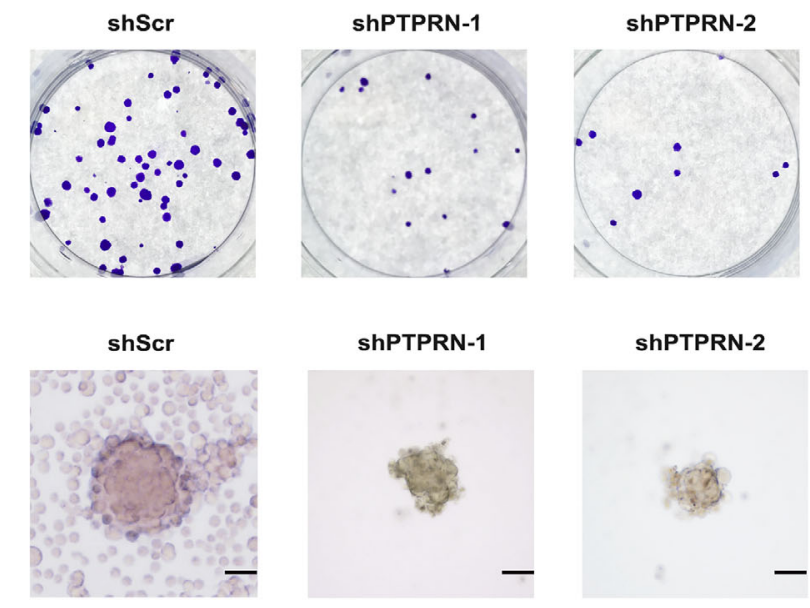

ShPTPRN-1
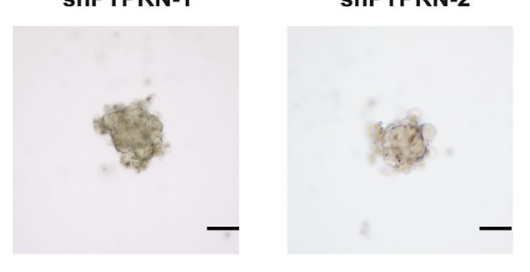

$\mathbf{F}$
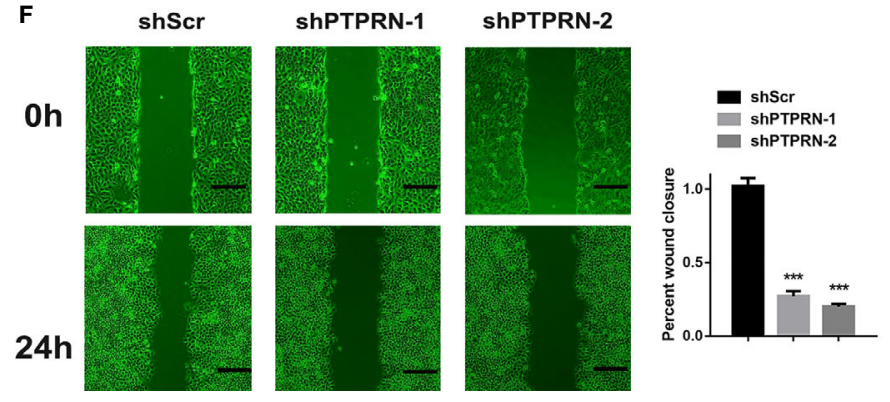
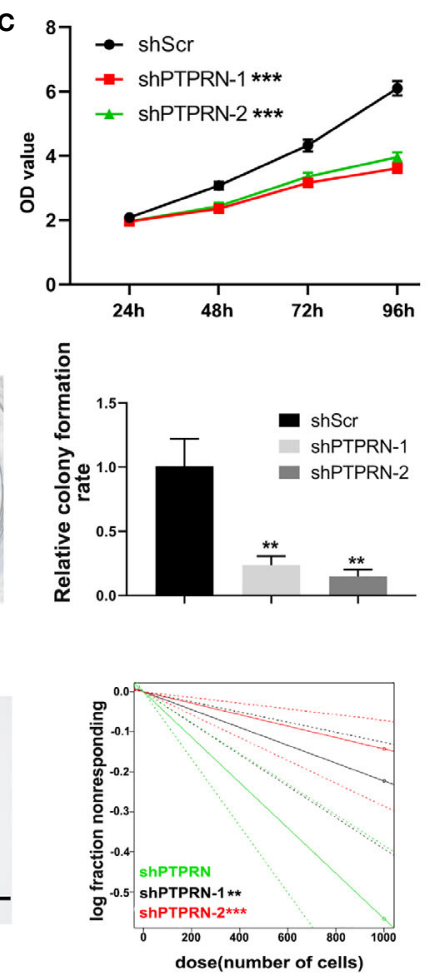

G

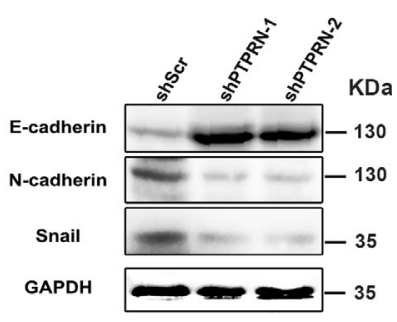

FIGURE 2 | PTPRN downregulation reduced the proliferation and migration of U87 cells. (A, B) The level of PTPRN was assayed by RT-qPCR and western blot analysis after transfection with PTPRN-shRNA; (C) The proliferation of the cells was detected by CCK-8; the corresponding OD values were detected at $24 \mathrm{~h}, 48 \mathrm{~h}$, $72 \mathrm{~h}$ and $96 \mathrm{~h}$; (D) The proliferation of the cells was detected by colony formation assays; the results were recorded using a camera at 14 days; (E) Sphere formation detected by microscope and calculated by ELDA after 14 days; (F) The migration of the cells detected by wound healing assay; the scratch was imaged at 0 and $24 \mathrm{~h}$; The area of scratch reduction was calculated; (G) EMT-related protein (E-cadherin, N-cadherin, and Snail) expression in shPTPRN U87 cells; scale bars, $100 \mu \mathrm{m} ;{ }^{* *} \mathrm{p}<0.01,{ }^{* \star *} \mathrm{p}<0.001$.

overexpression of PTPRN (Figure 6C). Treatment with BEZ235 reversed the growth and migration of PTPRN-overexpressing U251 glioma cells (Figures 6D-G).

\section{DISCUSSION}

Increasing evidence has shown that the PTP family does not always antagonize the activity of PTKs in regulating tyrosine phosphorylation and plays a crucial role in the initiation and progression of signalling cascades regulating cell functions, which cause many human diseases, such as cancer, metabolic syndrome and autoimmune diseases (12-14). The function and mechanism of action of PTPRN were investigated in certain tumors. These studies focused on the relationship between protein or mRNA expression and clinical prognosis, including hepatocellular carcinoma (15), lung cancer and other neuroendocrine tumors (16), ovarian cancer (17). In glioma research, Shergalis A et al. showed that PTPRN overexpression was strongly associated with poor overall survival in glioblastoma patients (18). Xu P et al. identified high PTPRN level as a crucial prognostic factor in glioma by weighted gene coexpression network analysis (WGCNA) (19). These study showed that PTPRN may play an important role in the occurrence and development of glioma; however, at present, there are no relevant experimental studies of PTPRN in vivo or in vitro in glioma. 
A

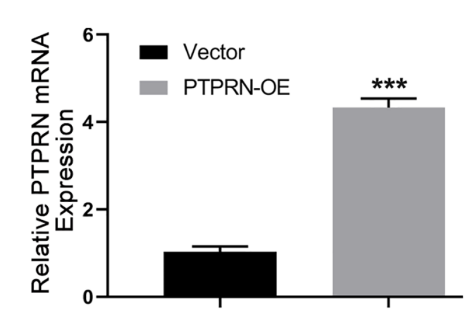

D

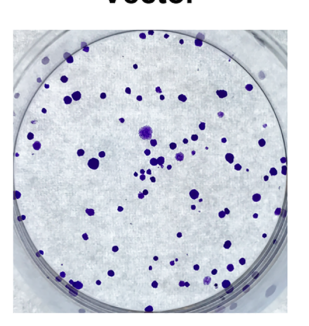

E

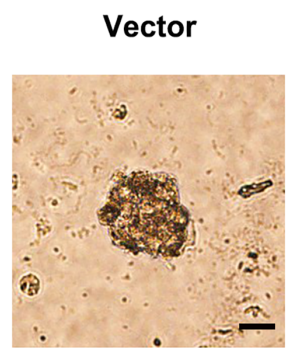

B

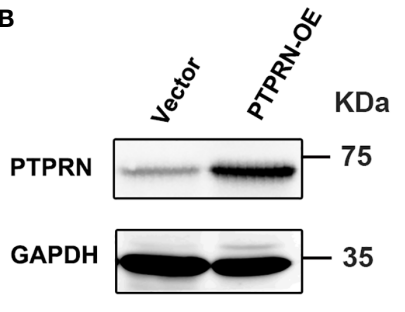

PTPRN-OE

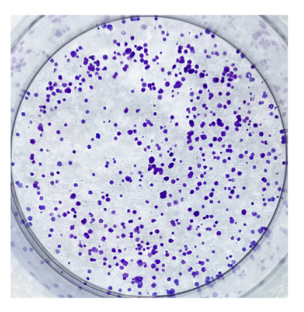

PTPRN-OE

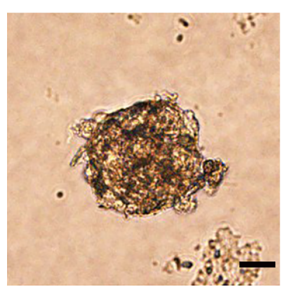

C
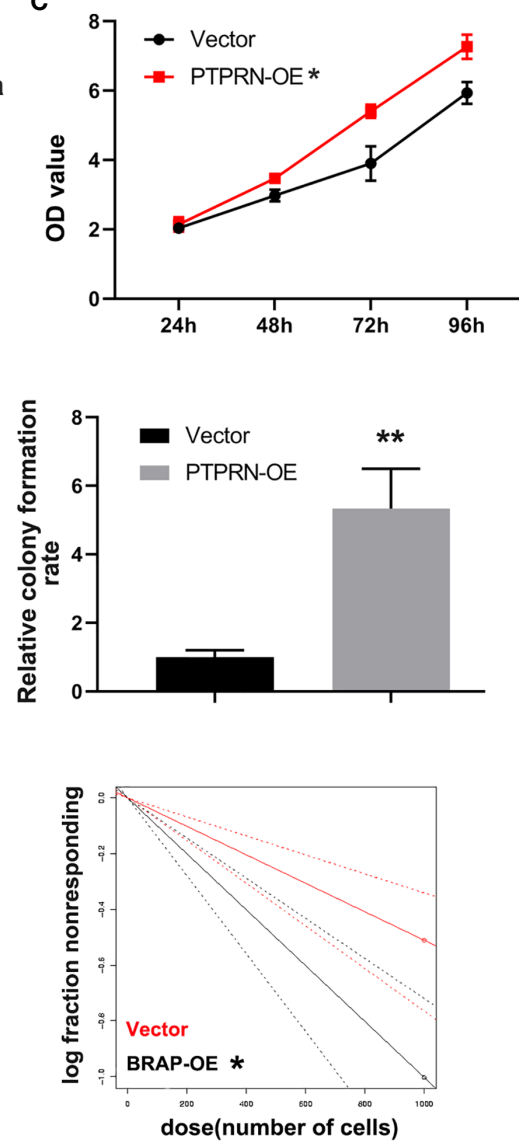

$\mathbf{F}$

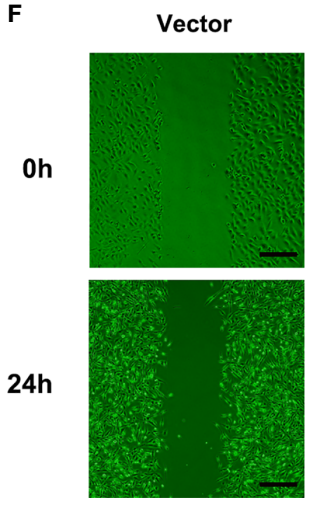

PTPRN-OE
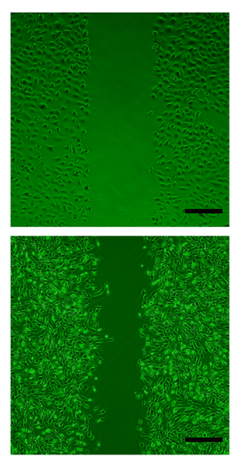

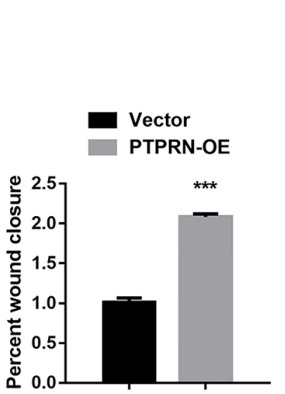

G

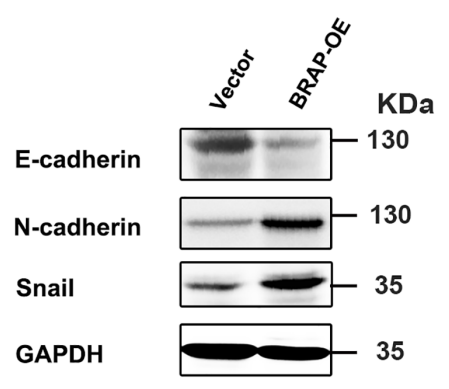

FIGURE 3 | PTPRN overexpression induced the proliferation and migration of U251 cells. (A, B) The level of PTPRN was assayed by RT-qPCR and western blot analysis after the transfection with PTPRN overexpression plasmid; (C) The proliferation of the cells was detected by CCK-8; the corresponding OD values were detected at 24 h, 48 h, 72 h, and 96 h; (D) The proliferation of the cells was detected by colony-formation assays; the results were recorded using a camera at 14 days; (E) Sphere formation detected by microscope and calculated by ELDA after 14 days; (F) Cell migration detected by wound healing assay; the scratch was imaged at 0 and $24 \mathrm{~h}$; The area of scratch reduction was calculated; (G) EMT-related protein (E-cadherin, N-cadherin, and Snail) expression in PTPRNoverexpression U251cells; scale bars, $100 \mu \mathrm{m} ;{ }^{\star} \mathrm{p}<0.05,{ }^{\star \star} \mathrm{p}<0.01,{ }^{\star \star *} \mathrm{p}<0.001$.

The results of the present study indicated that a low level of PTPRN was associated with an improved prognosis in glioma patients. The results of clinical experiments were further verified by cell phenotype experiment. PTPRN expression was closely related to MGMT. MGMT is a unique DNA repair enzyme. MGMT removed $\mathrm{O}^{6}-\mathrm{MG}$ from DNA, protect tumor cells from the damage of alkylating agents, and then lead to temozolomide resistance of tumor cells, which has become an 
A

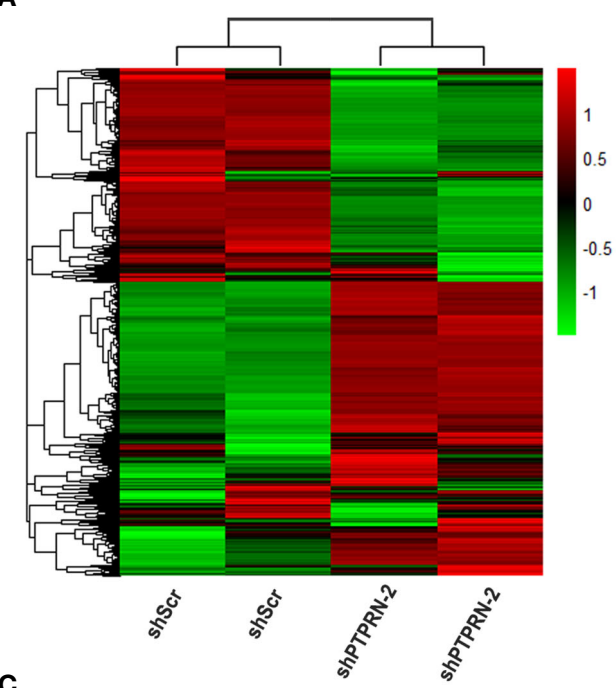

C

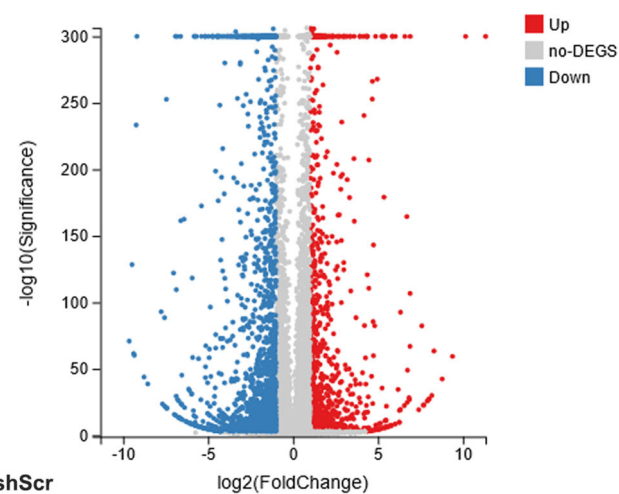

B

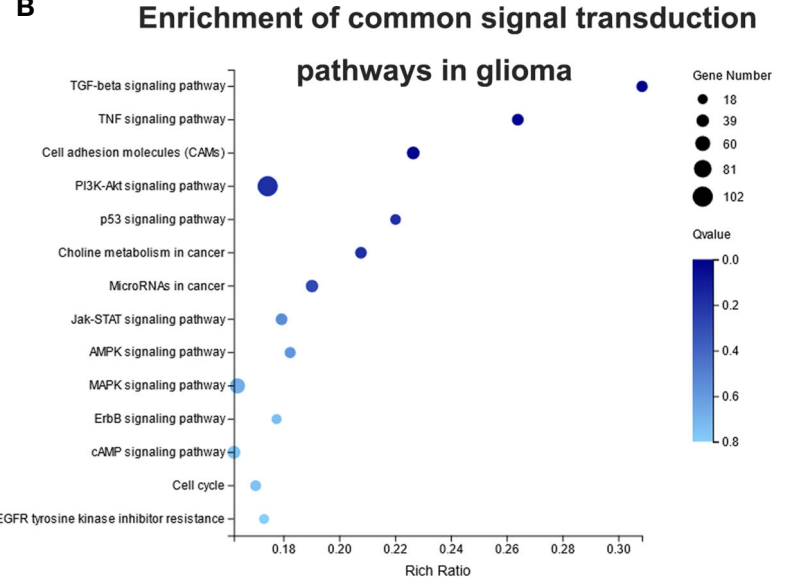

D
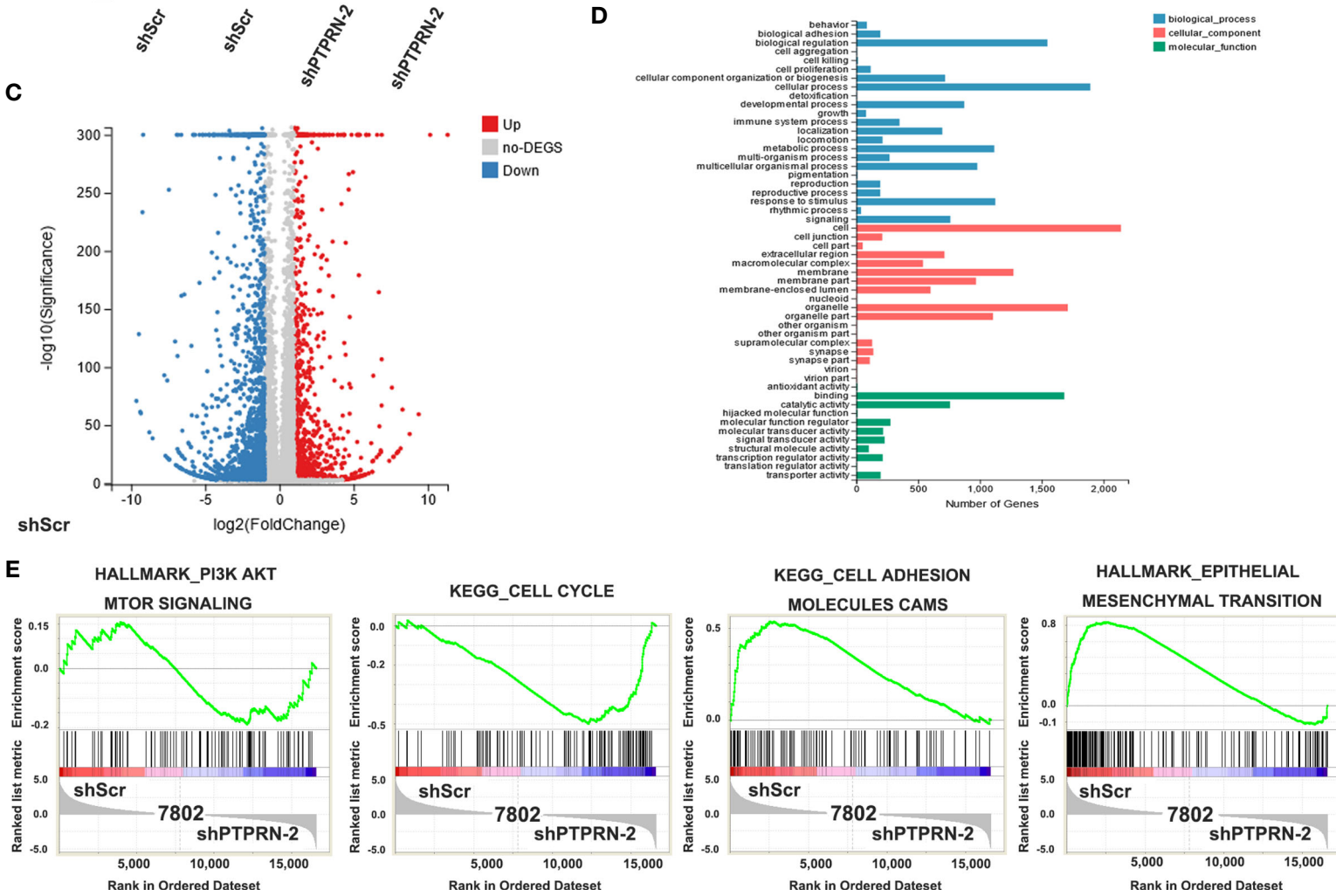

FIGURE 4 | Effect of PTPRN knockdown on the transcriptome of U87 glioma cells. (A) Heat map of differentially expressed genes in shPTPRN U87 glioma cells; red represents highly expressed genes, and the green represents genes with low expression; the expression level is directly proportional to the color depth; (B) the KEGG method was used to analyze the differential genes and to select the enrichment pathways; (C) Volcano plot analysis; red represents highly expressed genes, and blue represents genes with low expression; the expression level is directly proportional to the color depth; (D) Gene Ontology analysis showed differential genes were categorized into three groups, including biological process, cellular component and molecular function; (E) GSEA was used to select the enrichment pathways.

important factor restricting the effect of glioma chemotherapy. PI3K/AKT pathway is involved in the regulation of MGMT (20, 21). At present, the US Food and Drug Administration has granted a fast track for paxalisib, a PI3K inhibitor, to treat newly diagnosed glioblastoma patients with unmethylated MGMT gene promoter, who have completed preliminary radiotherapy and temozolomide treatment. Interestingly, we explored the mechanism by which PTPRN functions in glioma cells. GSEA showed that the PI3K/AKT pathway was also associated with the changes in PTPRN. The PI3K-Akt pathway is involved in the growth and apoptosis of cells. The activation of a variety of growth factors, hormones, and cytokines will lead to the further activation of this pathway, leading to the loss of normal differentiation and apoptosis of 
A

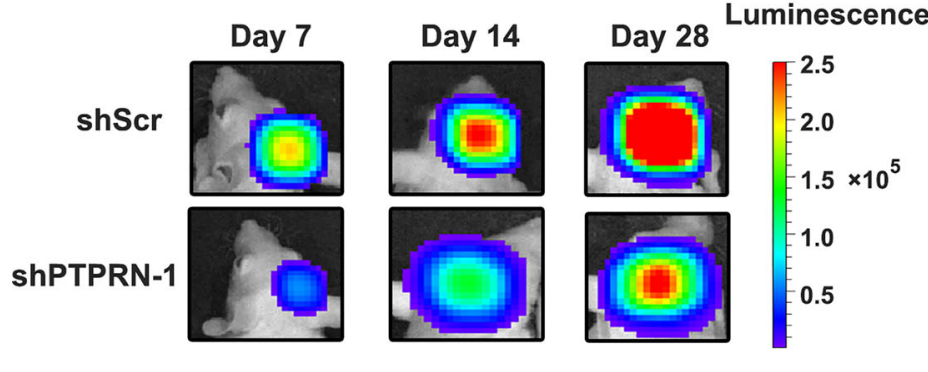

C

$$
\text { Day } 10
$$
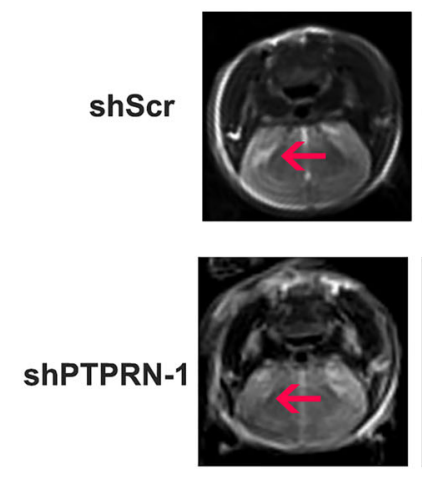

Day 20
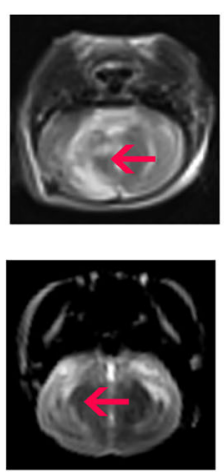

\section{Day 30}
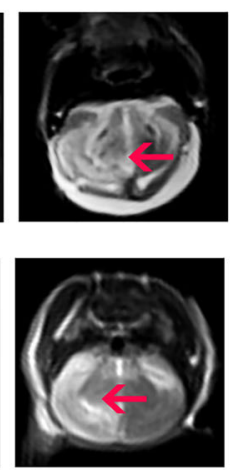

B

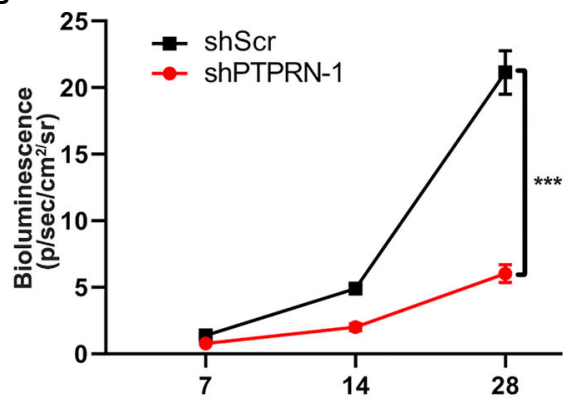

D

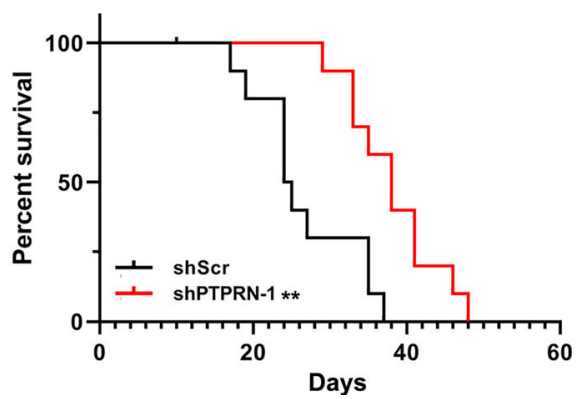

FIGURE 5 | PTPRN knockdown decreased tumor growth in a mouse xenograft model. (A) Representative image of the tumor volume measured by bioluminescence imaging; (B) The intensity of fluorescence penetration into the brain tumor; (C) Representative image of the tumor volume measured by MRI; (D) Survival analysis of PTPRN expression in 12 intracranially implanted nude mice; Kaplan-Meier survival curves were used to analysis the difference of prognosis between the control group and PTPRN knockdown group; ${ }^{* *} \mathrm{p}<0.01$, ${ }^{* \star *} \mathrm{p}<0.001$.

cells, leading to the occurrence of tumors (22). Notably, PI3K/ AKT induced the accumulation of $\beta$-catenin in the nucleus, upregulated the expression of the downstream genes, and promoted the progression of epithelial-mesenchymal transition (EMT) (23). Epithelial-mesenchymal transition (EMT) is a biological process in which epithelial cells transform into cells with mesenchymal phenotype through specific procedures. E-cadherin can maintain tight junctions between cells and prevent cell invasion, metastasis and diffusion. $\mathrm{N}$-cadherin is the main structural component of intercellular adhesion, and its main function is to mediate cell adhesion and migration. Snail, a transcription factor, binds to promoter regions such as E-cadherin to inhibit its transcription and promote epithelial-mesenchymal transition (24). In our experiment, PTPRN facilitated N-cadherin and Snail expression and inhibited E-cadherin expression, leading to tumor invasion and metastasis.

The results of a Co-IP experiment confirmed by mass spectrometry and Western blotting indicated that PTPRN regulated the activity of the PI3K/AKT pathway by interacting with HSP90AA1. HSP90AA1 is a member of the HSP90 family and one of the most important heat shock proteins. HSP90AA1 promotes the maturation, structural maintenance and proper regulation of specific target proteins and regulates cell cycle control and signal transduction. Some studies demonstrated that HSP90AA1 is closely related to a variety of malignant tumors (25, 26). A large-scale, multicentre, cross-validation clinical study demonstrated that plasma HSP $90 \alpha$ can be used as a pan-cancer biomarker (27). HSP90AA1 stabilizes and activates c-Myc and participates in the occurrence of colorectal carcinoma and hepatocellular carcinoma $(28,29)$. Xiao X et al. demonstrated that HSP90AA1 promotes autophagy through the PI3K/Akt/ mTOR pathway in osteosarcoma (30). Song $\mathrm{KH}$ et al. demonstrated that Nanog-activated HSP90A/AKT pathway is a potential target for immune refractory tumors (31). Therefore, we hypothesized that PTPRN interacts with HSP90AA1 to partially activate the PI3K/AKT pathway. The PI3K inhibitor treatment recovered PI3K/AKT pathway activity and phenotype of cells with PTPRN knockdown. These findings confirmed that the HSP90AA1/Akt signalling pathway may be involved in the proliferation and metastasis of glioma induced by PTPRN.

Thus, we suggest that glioma patients with high expression of PTPRN have a poor prognosis. PTPRN is an important proliferation- and metastasis-promoting factor. Furthermore, PTPRN may partially activate the PI3K/Akt pathway to influence the cell phenotype through HSP90AA1. These results indicate that reducing the expression of PTPRN in glioma cells can be used as a potential therapeutic strategy. 


\begin{tabular}{|l|c|c|c|}
\hline Protein & Mass [kDa] & Peptides & Score \\
\hline ENO1 & 47.1 & 8 & 435.36 \\
\hline CCT8 & 59.6 & 16 & 430.75 \\
\hline IDH2 & 50.9 & 6 & 429.71 \\
\hline HSP90AA1 & 68.3 & 13 & 427.62 \\
\hline HSD17B10 & 26.9 & 11 & 378.54 \\
\hline EEF2 & 95.3 & 19 & 377.51 \\
\hline STK38 & 54.2 & 19 & 367.02 \\
\hline
\end{tabular}

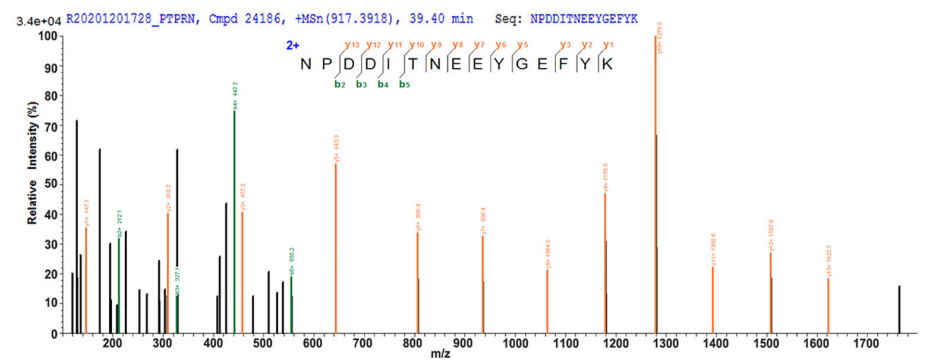

C

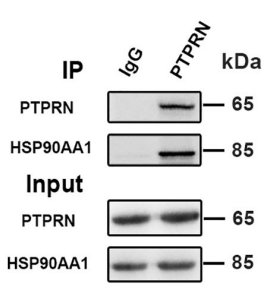

D

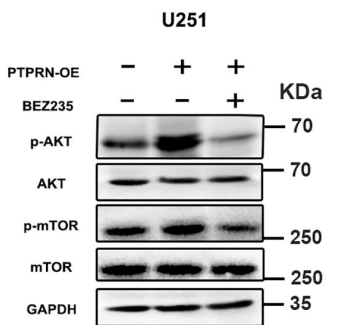

E

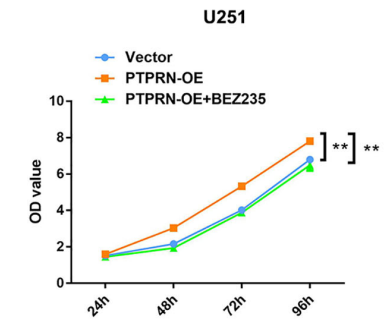

$\mathbf{F}$

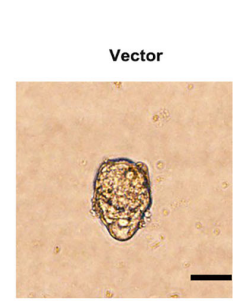

U251

PTPRN-OE
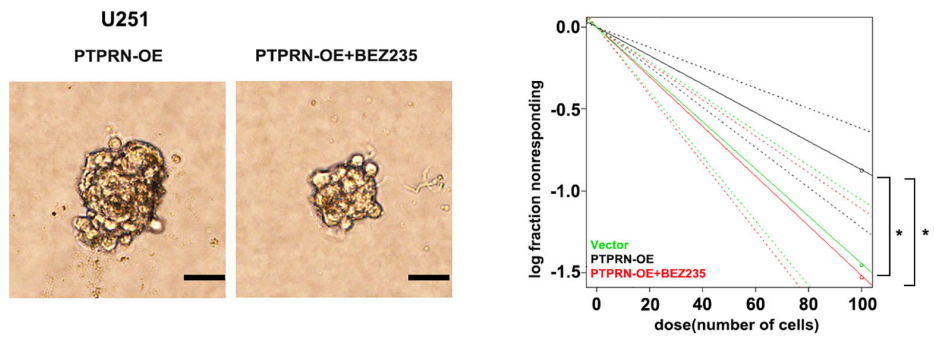

G

U251

Vector

PTPRN-OE

PTPRN-OE+BEZ235

oh
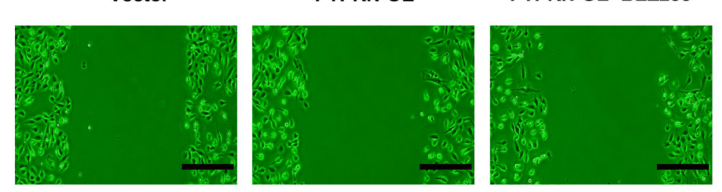

24h
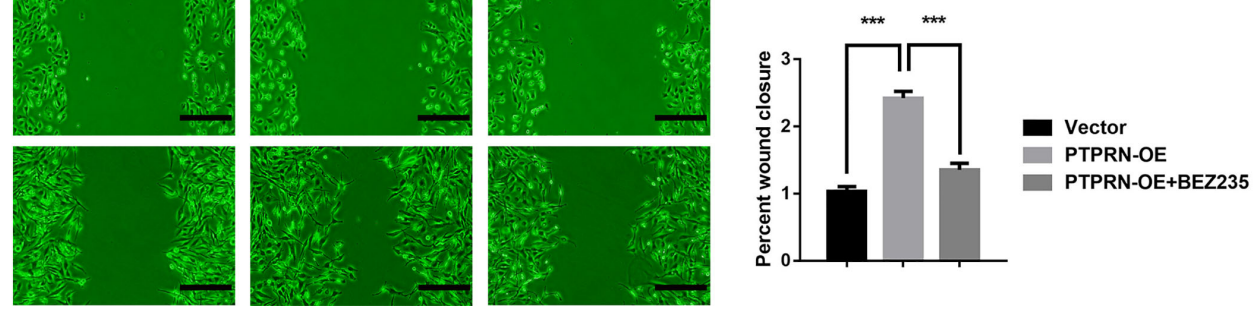

FIGURE 6 | PTPRN activated the PI3K/AKT pathway by interacting with heat shock protein 90 alpha family class A member 1 (HSP90AA1). (A) Differentially expressed proteins interacting with PTPRN were identified by Co-IP and LC-MS/MS from FLAG-HA-PTPRN-expressing 293T cells, including an interaction between PTPRN and HSP90AA1. (B) Secondary spectrogram of target peptide showed PTPRN bind with HSP90AA1 directly. (C) Immunoprecipitation and western blotting demonstrated that PTPRN interacted with HSP90AA1 in U87 cells; (D) BEZ235 was used to reverse PI3K/AKT pathway activity in U251 cells with overexpression of PTPRN. AKT, pAKT, mTOR, and p-mTOR expression were shown by western blot; (E) Cell proliferation detected by CCK-8; the corresponding OD values were detected at 24 h, 48 h, $72 \mathrm{~h}$ and $96 \mathrm{~h}$; (F) Sphere formation detected by microscope and calculated by ELDA after 14 days; (G) The migration of the cells detected by wound healing assay; the scratch was imaged at 0 and $24 \mathrm{~h}$; The area of scratch reduction was calculated; scale bars, $100 \mu \mathrm{m} ;{ }^{\star} \mathrm{p}<0.05,{ }^{\star \star *} \mathrm{p}<0.01,{ }^{\star \star \star} \mathrm{p}<0.001$.

\section{DATA AVAILABILITY STATEMENT}

The datasets presented in this study can be found in online repositories. The names of the repository/repositories and accession number(s) can be found below: GEO Submission (GSE175831), https://www.ncbi.nlm.nih.gov/geo/query/acc.cgi? acc=GSE175831.

\section{ETHICS STATEMENT}

The studies involving human participants were reviewed and approved by Ethics Committee of Tianjin Huanhu Hospital. The patients/ participants provided their written informed consent to participate in this study. The animal study was reviewed and approved by Tianjin Huanhu hospital Animal Use and Care Committee. 


\section{AUTHOR CONTRIBUTIONS}

$\mathrm{DW}, \mathrm{FT}$, and XL carried out most of the experimental work and the analysis of data. YF and YZ completed parts of the pathological experiment and uploaded the GEO data. HZ, JZ, BC, and BW provided scientific and administrative oversight for the conduct of the research and revised the manuscript. All authors contributed to the article and approved the submitted version.

\section{FUNDING}

This work was supported by Chinese National Natural Science Foundation [grant number 82103247, 82171359, 81902477]; Tianjin Jinnan District Science and technology plan project [grant number 20200110]; Beijing Tianjin Hebei basic research cooperation project [grant number 19JCZDJC64600(Z)]; Science

\section{REFERENCES}

1. Ostrom QT, Gittleman H, Stetson L, Virk S, Barnholtz-Sloan JS. Epidemiology of Intracranial Gliomas. Prog Neurol Surg (2018) 30:1-11. doi: 10.1159/000464374

2. Weller M, Wick W, Aldape K, Brada M, Berger M, Pfister SM, et al. Glioma. Nat Rev Dis Primers (2015) 1:15017. doi: 10.1038/nrdp.2015.17

3. Xie J, Zhang B, Lan MS, Notkins AL. Genomic Structure and Promoter Sequence of the Insulin-Dependent Diabetes Mellitus Autoantigen, IA-2 (PTPRN). Genomics (1998) 54(2):338-43. doi: 10.1006/geno.1998.5583

4. Solimena M, Dirkx RJr., Hermel JM, Pleasic-Williams S, Shapiro JA, Caron L, et al. ICA 512, an Autoantigen of Type I Diabetes, Is an Intrinsic Membrane Protein of Neurosecretory Granules. EMBO J (1996) 15(9):2102-14. doi: 10.1002/j.1460-2075.1996.tb00564.x

5. Hermel JM, Dirkx RJr., Solimena M. Post-Translational Modifications of ICA512, a Receptor Tyrosine Phosphatase-Like Protein of Secretory Granules. Eur J Neurosci (1999) 11(8):2609-20. doi: 10.1046/j.14609568.1999.00677.x

6. Torii S. Expression and Function of IA-2 Family Proteins, Unique Neuroendocrine-Specific Protein-Tyrosine Phosphatases. Endocr J (2009) 56(5):639-48. doi: 10.1507/endocrj.k09e-157

7. Torii S, Saito N, Kawano A, Hou N, Ueki K, Kulkarni RN, et al. Gene Silencing of Phogrin Unveils its Essential Role in Glucose-Responsive Pancreatic Beta-Cell Growth. Diabetes (2009) 58(3):682-92. doi: 10.2337/ db08-0970

8. Louraki M, Katsalouli M, Kanaka-Gantenbein C, Kafassi N, Critselis E, Kallinikou D, et al. The Prevalence of Early Subclinical Somatic Neuropathy in Children and Adolescents With Type 1 Diabetes Mellitus and its Association With the Persistence of Autoantibodies to Glutamic Acid Decarboxylase (GAD) and Islet Antigen-2 (IA-2). Diabetes Res Clin Pract (2016) 117:82-90. doi: 10.1016/j.diabres.2016.04.044

9. Krischer JP, Cuthbertson DD, Yu L, Orban T, Maclaren N, Jackson R, et al. Screening Strategies for the Identification of Multiple Antibody-Positive Relatives of Individuals With Type 1 Diabetes. J Clin Endocrinol Metab (2003) 88(1):103-8. doi: 10.1210/jc.2002-020760

10. Cui Y, Chen W, Chi J, Wang L. Differential Expression Network Analysis for Diabetes Mellitus Type 2 Based on Expressed Level of Islet Cells. Ann Endocrinol (Paris) (2016) 77(1):22-9. doi: 10.1016/j.ando.2015. 11.002

11. Mziaut H, Trajkovski M, Kersting S, Ehninger A, Altkruger A, Lemaitre RP, et al. Synergy of Glucose and Growth Hormone Signalling in Islet Cells Through ICA512 and STAT5. Nat Cell Biol (2006) 8(5):435-45. doi: 10.1038/ ncb1395

12. Pulido R, Stoker AW, Hendriks WJ. PTPs Emerge as PIPs: Protein Tyrosine Phosphatases With Lipid-Phosphatase Activities in Human Disease. Hum Mol Genet (2013) 22(R1):R66-76. doi: 10.1093/hmg/ddt347 and Technology Development Foundation of Henan Province [212102310115, 212102310135]; Henan Provincial Medical Science and Technology Project [SBGJ202002025, SBGJ202003009]; Henan Medical Science and Technology Innovation Talent Project [YXKC2020045].

\section{SUPPLEMENTARY MATERIAL}

The Supplementary Material for this article can be found online at: https://www.frontiersin.org/articles/10.3389/fonc.2021.676287/ full\#supplementary-material

Supplementary Figure 1 | The expression of VEGF and MGMT by immunohistochemistry staining in high-grade glioma. The expression of VEGF and MGMT detected by immunohistochemistry staining in high-grade glioma (negative and positive).

13. Jiang ZX, Zhang ZY. Targeting PTPs With Small Molecule Inhibitors in Cancer Treatment. Cancer Metastasis Rev (2008) 27(2):263-72. doi: 10.1007/ s10555-008-9113-3

14. Solouki S, August A, Huang W. Non-Receptor Tyrosine Kinase Signaling in Autoimmunity and Therapeutic Implications. Pharmacol Ther (2019) 201:3950. doi: 10.1016/j.pharmthera.2019.05.008

15. Zhangyuan G, Yin Y, Zhang W, Yu W, Jin K, Wang F, et al. Prognostic Value of Phosphotyrosine Phosphatases in Hepatocellular Carcinoma. Cell Physio Biochem (2018) 46(6):2335-46. doi: 10.1159/000489625

16. Xie H, Notkins AL, Lan MS. IA-2, a Transmembrane Protein Tyrosine Phosphatase, is Expressed in Human Lung Cancer Cell Lines With Neuroendocrine Phenotype. Cancer Res (1996) 56(12):2742-4.

17. Bauerschlag DO, Ammerpohl O, Brautigam K, Schem C, Lin Q, Weigel MT, et al. Progression-Free Survival in Ovarian Cancer is Reflected in Epigenetic DNA Methylation Profiles. Oncology (2011) 80(1-2):12-20. doi: 10.1159/ 000327746

18. Shergalis A, Bankhead A3rd, Luesakul U, Muangsin N, Neamati N. Current Challenges and Opportunities in Treating Glioblastoma. Pharmacol Rev (2018) 70(3):412-45. doi: 10.1124/pr.117.014944

19. Xu P, Yang J, Liu J, Yang X, Liao J, Yuan F, et al. Identification of Glioblastoma Gene Prognosis Modules Based on Weighted Gene Co-Expression Network Analysis. BMC Med Genomics (2018) 11(1):96. doi: 10.1186/s12920-018-0407-1

20. Wick W, Weller M, van den Bent M, Sanson M, Weiler M, von Deimling A, et al. MGMT Testing-the Challenges for Biomarker-Based Glioma Treatment. Nat Rev Neurol (2014) 10(7):372-85. doi: 10.1038/nrneurol.2014.100

21. Hegi ME, Liu L, Herman JG, Stupp R, Wick W, Weller M, et al. Correlation of O6-Methylguanine Methyltransferase (MGMT) Promoter Methylation With Clinical Outcomes in Glioblastoma and Clinical Strategies to Modulate MGMT Activity. J Clin Oncol (2008) 26(25):4189-99. doi: 10.1200/ JCO.2007.11.5964

22. Polivka JJr., Janku F. Molecular Targets for Cancer Therapy in the PI3K/AKT/ mTOR Pathway. Pharmacol Ther (2014) 142(2):164-75. doi: 10.1016/ j.pharmthera.2013.12.004

23. Revathidevi S, Munirajan AK. Akt in Cancer: Mediator and More. Semin Cancer Biol (2019) 59:80-91. doi: 10.1016/j.semcancer.2019.06.002

24. Lamouille S, Xu J, Derynck R. Molecular Mechanisms of EpithelialMesenchymal Transition. Nat Rev Mol Cell Biol (2014) 15(3):178-96. doi: $10.1038 / \mathrm{nrm} 3758$

25. Elzakra N, Cui L, Liu T, Li H, Huang J, Hu S. Mass Spectrometric Analysis of SOX11-Binding Proteins in Head and Neck Cancer Cells Demonstrates the Interaction of SOX11 and HSP90alpha. J Proteome Res (2017) 16(11):3961-8. doi: 10.1021/acs.jproteome.7b00247

26. Dong Z, Yang P, Qiu X, Liang S, Guan B, Yang H, et al. KCNQ1OT1 Facilitates Progression of non-Small-Cell Lung Carcinoma via Modulating miRNA-27b-3p/HSP90AA1 Axis. J Cell Physiol (2019) 234(7):11304-14. doi: $10.1002 /$ jcp. 27788 
27. Liu W, Li J, Zhang P, Hou Q, Feng S, Liu L, et al. A Novel Pan-Cancer Biomarker Plasma Heat Shock Protein 90alpha and its Diagnosis Determinants in Clinic. Cancer Sci (2019) 110(9):2941-59. doi: 10.1111/cas.14143

28. Shi W, Feng L, Dong S, Ning Z, Hua Y, Liu L, et al. FBXL6 Governs C-MYC to Promote Hepatocellular Carcinoma Through Ubiquitination and Stabilization of HSP90AA1. Cell Comm Signaling CCS (2020) 18(1):100. doi: 10.1186/ s12964-020-00604-y

29. Tang J, Yan T, Bao Y, Shen C, Yu C, Zhu X, et al. LncRNA GLCC1 Promotes Colorectal Carcinogenesis and Glucose Metabolism by Stabilizing C-Myc. Nat Commun (2019) 10(1):3499. doi: 10.1038/s41467-019-11447-8

30. Xiao X, Wang W, Li Y, Yang D, Li X, Shen C, et al. HSP90AA1-Mediated Autophagy Promotes Drug Resistance in Osteosarcoma. J Exp Clin Cancer Res (2018) 37(1):201. doi: 10.1186/s13046-018-0880-6

31. Song KH, Oh SJ, Kim S, Cho H, Lee HJ, Song JS, et al. HSP90A Inhibition Promotes Anti-Tumor Immunity by Reversing Multi-Modal Resistance and Stem-Like Property of Immune-Refractory Tumors. Nat Commun (2020) 11 (1):562. doi: 10.1038/s41467-019-14259-y
Conflict of Interest: The authors declare that the research was conducted in the absence of any commercial or financial relationships that could be construed as a potential conflict of interest.

Publisher's Note: All claims expressed in this article are solely those of the authors and do not necessarily represent those of their affiliated organizations, or those of the publisher, the editors and the reviewers. Any product that may be evaluated in this article, or claim that may be made by its manufacturer, is not guaranteed or endorsed by the publisher.

Copyright $\odot 2021$ Wang, Tang, Liu, Fan, Zheng, Zhuang, Chen, Zhuo and Wang. This is an open-access article distributed under the terms of the Creative Commons Attribution License (CC BY). The use, distribution or reproduction in other forums is permitted, provided the original author(s) and the copyright owner $(s)$ are credited and that the original publication in this journal is cited, in accordance with accepted academic practice. No use, distribution or reproduction is permitted which does not comply with these terms. 